\title{
METROLOGÍA Y ARQUITECTURA MODULAR EN EL PUERTO DE LA PICOLA (SANTA POLA, ALICANTE) AL FINAL DEL SIGLO V a.C.
}

\author{
POR \\ PIERRE MORET y ALAIN BADIE \\ Unité Toulousaine d'Archéologie et d'Histoire
}

\section{RESUMEN}

El puerto fortificado de La Picola (Santa Pola), situado en la costa alicantina al Sureste de Elche, fue construido hacia 430 a.C. y abandonado al cabo de un siglo. El estudio de las estructuras conservadas demuestra que se utilizó la misma unidad de medida - un pie de 29,7 a $30 \mathrm{~cm}$ - en todas las etapas del programa de construcción, desde la fabricación de los adobes hasta las grandes divisiones del plano regulador. La trama de las calles y de las manzanas, organizada en torno a un eje de simetría, se basa en un módulo de 5 brazas $(5 \times 6$ pies $)$, mientras que la anchura de las casas sigue un módulo de 2 brazas $(2 \times 6$ pies $)$. El origen de este esquema moduar es probablemente griego.

\section{SUMMARY}

La Picola (Santa Pola, Alicante) is a fortified port to the south-east of Elche. Built c. $430 \mathrm{BC}$, it was abandoned within a hundred years. The surviving structures indicate that the same unit of measure was employed at every stage in the building of the port. This foot -equivalent to between 29,7 and $30 \mathrm{~cm}$ - is the basis of everything from mud bricks to the settlement's ground-plan. The network of streets and buildings is organised symmetrically, using a five-fathom or 30foot division; houses are two fathoms or 12 feet wide. This type of modular organisation is probably Greek in origin.

El yacimiento arqueológico de La Picola está situado al pie de la Sierra de Santa Pola, entre el casco urbano del puerto de Santa Pola y una extensa área de salinas y lagunas que conectan con la desembocadura del río Vinalopó (fig. 1).

Después de dos excavaciones de urgencia, en 1977 y 1989, María José Sánchez, directora del Museo arqueológico de Santa Pola, impulsó un estudio programado del yacimiento. De 1991 a 1995, un equipo hispano-francés dirigido por P. Rouillard, M.J. Sánchez y P. Sillières llevó a cabo cuatro campañas de excavación ${ }^{1}$. Se documentaron dos momentos de ocupación, separados por una larga fase de abandono. Las estructuras más recientes pertenecen a un

\footnotetext{
${ }^{1}$ Con el respaldo de la Conselleria de Cultura de la Generalitat Valenciana, el Ayuntamiento de Santa Pola, el Ministerio francés de Asuntos Exteriores y la Casa de Velázquez.
}

conjunto de factorías de salazón romanas, activas entre fines del siglo I a.C. y fines del siglo Iv d.C. La primera ocupación del lugar, en época ibérica, corresponde a un pequeño puerto fortificado estrechamente vinculado con la ciudad de Ilici (La Alcudia de Elche), que estaba situada $14 \mathrm{~km}$ tierra adentro. La gran variedad de cerámicas áticas (copas, cráteras, ánforas y contenedores de perfume) que aparecieron en los niveles de ocupación y de destrucción del asentamiento ibérico, permite fechar su construcción hacia 450/430 a.C. y su abandono al cabo de un siglo, hacia 330 a.C. ${ }^{2}$. El propósito de esta contribución es estudiar el esquema de este poblado, cuya regularidad no tiene parangón en el panorama arqueológico de la Iberia de los siglos v y Iv a.C. ${ }^{3}$.

\section{LOS DATOS ARQUITECTÓNICOS}

Durante el siglo que duró su existencia, el asentamiento de La Picola no experimentó cambios importantes en cuanto a su urbanismo. Las únicas transformaciones que se han detectado atañen a la subdivisión interna de las manzanas. Fuera de estas reformas puntuales, podemos afirmar que la planta trazada hacia 430 a.C., en el momento de su fundación, no ha sido modificada.

Sin embargo, nuestro conocimiento del poblado ibérico dista mucho de ser completo. La destrucción del yacimiento estaba ya muy avanzada cuando empezaron las excavaciones arqueológicas. Las rotura-

\footnotetext{
${ }^{2}$ Una reseña breve de las tres primeras campañas puede ser consultada en P. Moret, A. Puigcerver, P. Rouillard, M.J. SÁNCHEZ y P. Sillières, «The Fortified Settlement of La Picola (Santa Pola, Alicante) and the Greek Influence in South-east Spain», en Social Complexity and the Development of Towns in Iberia (Proceedings of the British Academy, 86), Londres, 1995, 109-125, en espera de la publicación monográfica definitiva (P. RouILlaRd et al., Le site antique de La Picola à Santa Pola (Alicante), en prensa).

${ }^{3}$ Los autores quieren expresar su deuda con Pierre Sillières, Pierre Rouillard y Eric Gailledrat. Este trabajo no hubiera sido posible sin su estrecha colaboración.
} 


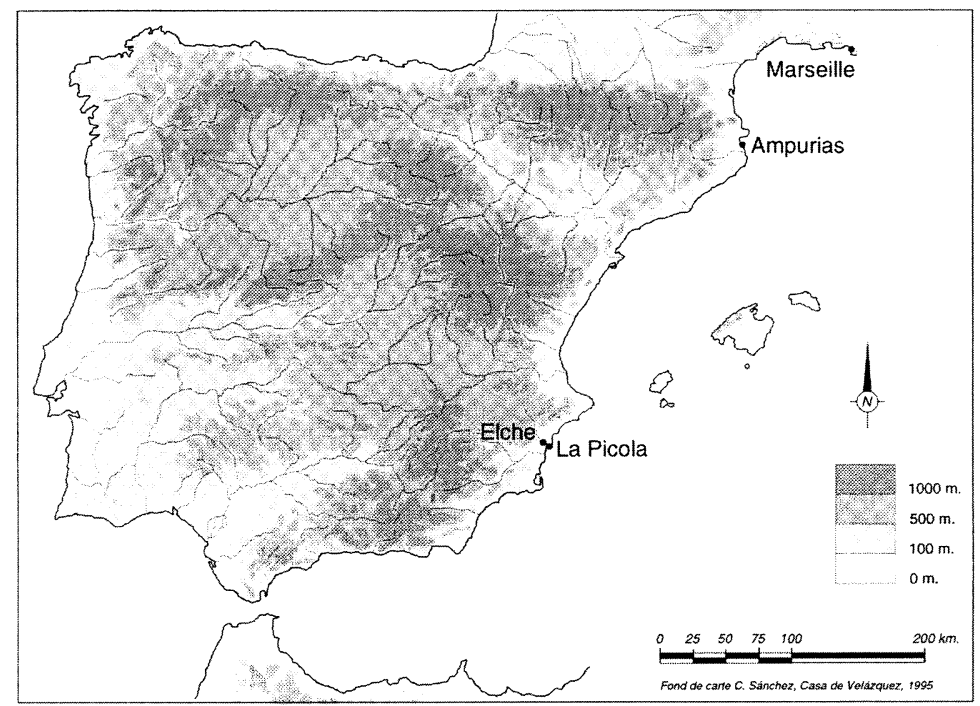

Fig. 1.-Localización del yacimiento de La Picola.

ciones y los aterrazamientos agrícolas de época moderna (siglos XVIII y XIX) han acarreado un arrasamiento general del terreno y la destrucción completa de los estratos antiguos en dos terceras partes del yacimiento. A esto se añadieron, en época más reciente, varias trincheras de alcantarillado que atraviesan el yacimiento de lado a lado. La estrategia de excavación tuvo que adaptarse a estas circunstancias adversas, recurriendo a sondeos profundos en los sectores más alterados, donde el foso perimetral del recinto ibérico es la única estructura antigua que se ha conservado. Con esta documentación truncada, no es de extrañar que no podamos proponer más que una reconstrucción parcial de la planta del poblado. Aun así, los datos son suficientes, como veremos, para intentar una valoración del proyecto arquitectónico tan peculiar que se plasmó en La Picola.

\section{EL SISTEMA DEFENSIVO}

El puerto ibérico de La Picola estaba protegido por una muralla de forma romboidal, casi cuadrada ${ }^{4}$

${ }^{4}$ Véase P. MoRet, Les fortifications ibériques, de la fin de l'âge du bronze à la conquête romaine, Madrid, Casa de Velázquez, 1996, 214-216 y 489-490.

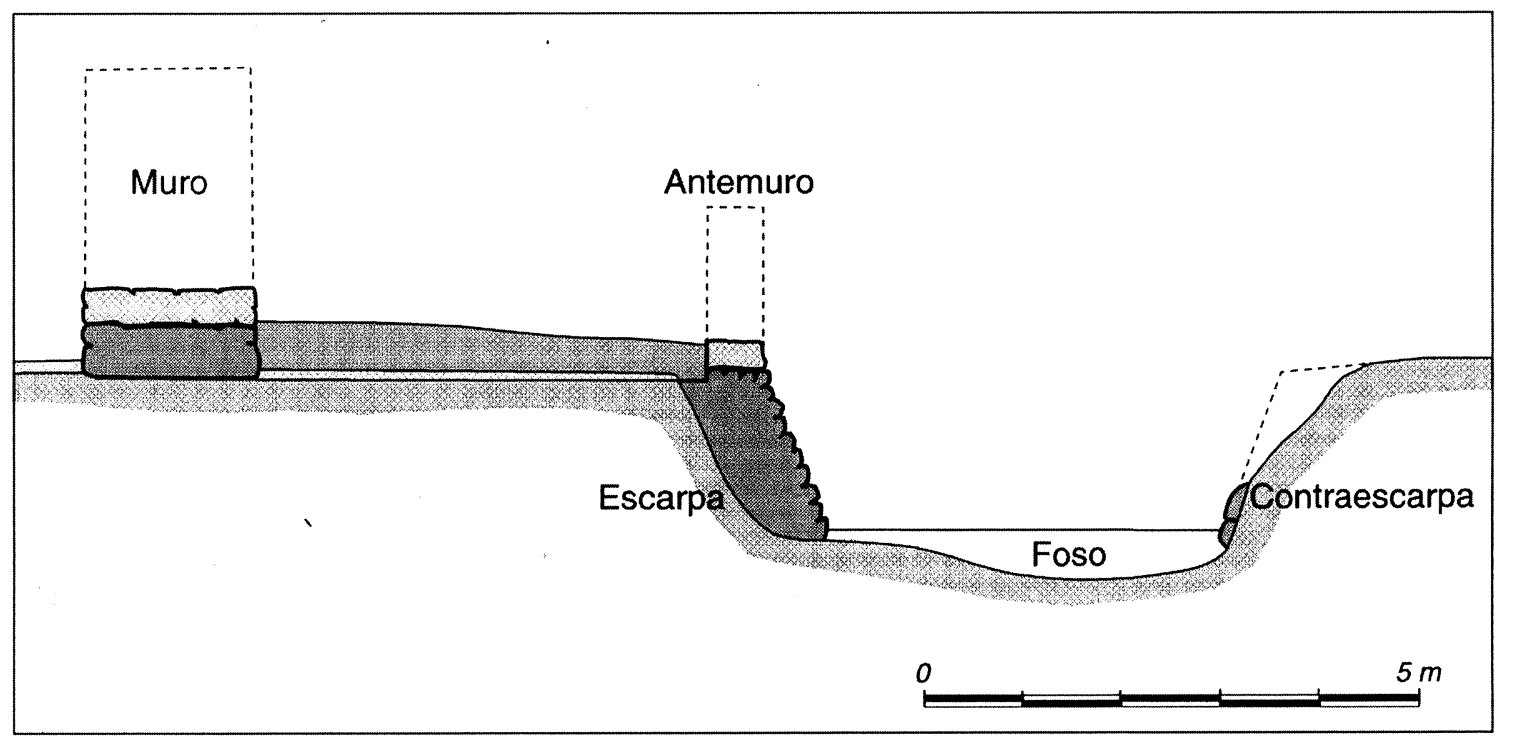

Fig. 2.-Perfil reconstruido del sistema defensivo. 


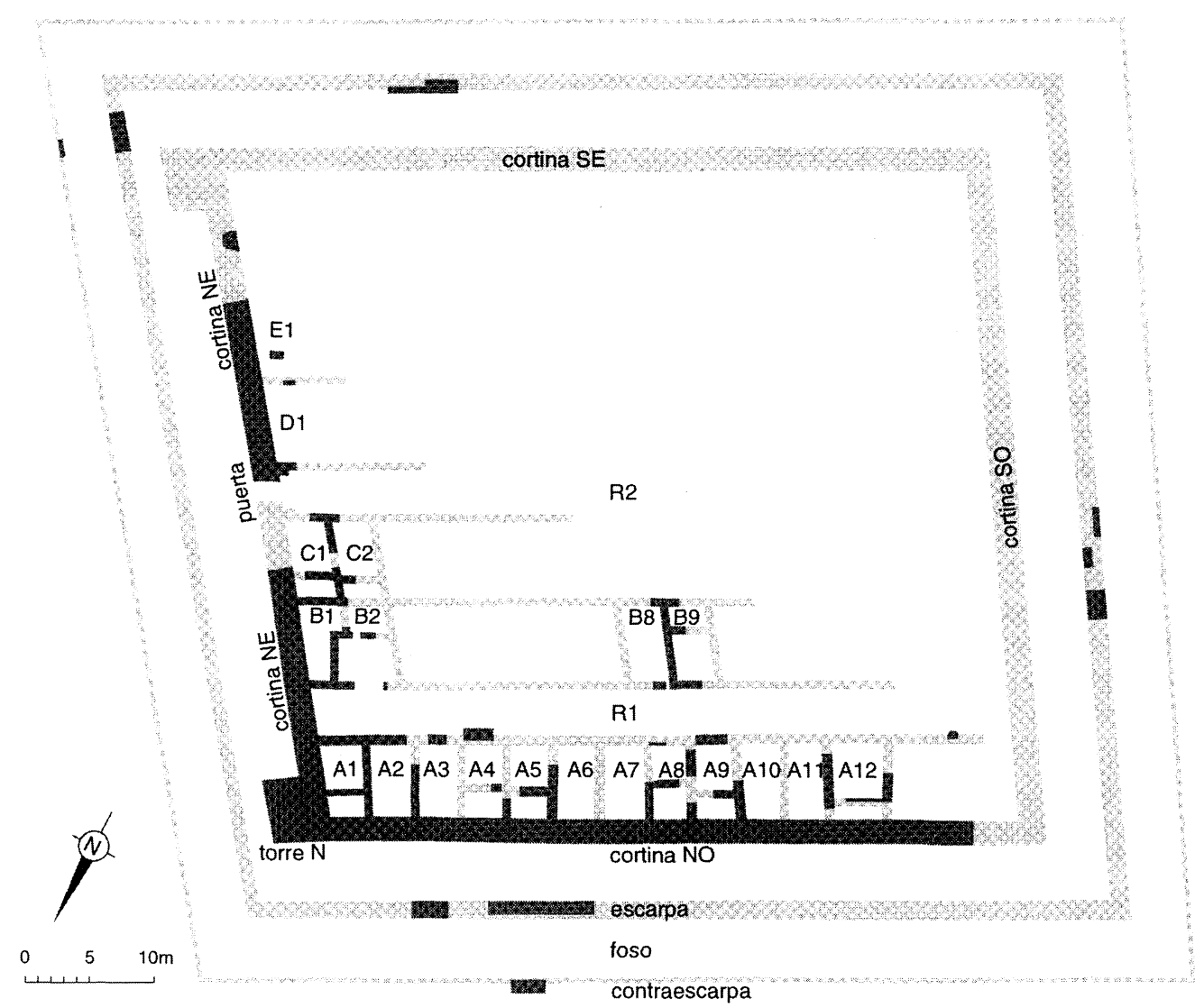

Fig. 3.-Plano de las estructuras de época ibérica. Gris oscuro, estructuras conservadas; gris claro, estructuras restituidas.

(fig. 3). Su área superficial es pequeña: $3.350 \mathrm{~m}^{2}$ intra muros, $6.100 \mathrm{~m}^{2}$ incluyendo el foso. Se localizó una puerta en el centro de la cortina noreste, del lado opuesto al mar. Estaba protegida por la torre angular norte $\mathrm{y}$, probablemente, por otra torre simétrica en el ángulo este. El sistema defensivo, en conjunto, ocupa una banda de terreno de 12 a $13 \mathrm{~m}$ de ancho. Lo constituyen cinco elementos sucesivos: una muralla, un glacis, una escarpa rematada por un antemuro, un foso y una contraescarpa (fig. 2).

Las cortinas se han conservado tan sólo en los lados noreste (longitud: $56 \mathrm{~m}$ ) y noroeste. Son rectas y su anchura varía entre 1,65 y 1,80 m. Están constituidas por un zócalo bajo $(0,75 \mathrm{~m})$ de piedras sobre el que se alzaba un muro de adobes. Un foso paralelo a la muralla, separado de esta última por un glacis o explanada de 5 a $5,5 \mathrm{~m}$ de ancho, rodeaba el asentamiento en sus cuatro lados. Su profundidad era de $2 \mathrm{~m}$, su anchura de 4 a $5 \mathrm{~m}$ en el fondo y de 5 a $6 \mathrm{~m}$ al nivel del suelo. Sendos paramentos de piedra en talud formaban la escarpa y la contraescarpa. Último elemento de este complejo sistema defensivo, el antemuro que hacía barrera encima del muro de escarpa era de adobes y su grosor apenas alcanzaba los 70 centímetros.

\section{MANZANAS Y CALLES}

Las estructuras de hábitat están peor conservadas que las fortificaciones. Sólo en el sector norte, a lo largo de las cortinas noreste y noroeste, pudo ser documentado el esquema interior del poblado (fig. 3). Una primera fila de casas, que llamaremos fila $A$, estaba adosada a la cortina noroeste. La calle $\mathrm{R}$ 1 separaba la fila $\mathrm{A}$ de la fila $\mathrm{B}$, la cual se conserva parcialmente en un tramo de treinta metros a partir de la cortina noreste. La fila $\mathrm{C}$ estaba pegada a la fila $\mathrm{B}$, formando con ella un solo bloque cuyas casas se abrían, respectivamente, a la calle $\mathrm{R} 2$ y a la calle R 1. La calle R 2, que corría por el medio del poblado, desembocaba en la puerta noreste del recinto. Los indicios se vuelven mucho más escasos en la mitad sureste del yacimiento. Lo único que se puede decir es que se puede atribuir varios restos de muros y de suelos, hallados cerca de la cortina noreste, a una manzana simétrica de B-C. 
Las filas estaban divididas en compartimientos rectangulares por medio de una serie de muros paralelos entre sí ${ }^{5}$. La fila $\mathrm{A}$, conservada en casi toda su longitud, incluía al menos 13 compartimientos. A su vez, la mayoría de estos compartimientos estaban divididos por un tabique en dos habitaciones de tamaño desigual. La primera estancia, que se abre a la calle, ocupa en términos medios los dos tercios del compartimiento. Los muros interiores son de adobes sobre zócalo de piedras, como la muralla. El área superficial de los compartimientos, en promedio, es de $20 \mathrm{~m}^{2}$, y su longitud es, casi siempre, el doble de su anchura.

Evidentemente, no se descarta la posibilidad de que dos o más de dos compartimientos pudieran estar unidos para formar una casa más compleja. La mala conservación de los muros no ha permitido evidenciar la existencia de umbrales de puerta en las paredes laterales de los compartimientos. Sin embargo, hemos constatado que el compartimiento B 1 no tenía acceso desde la calle, por lo que consideramos probable que el conjunto B $1+\mathrm{B} 2$ formara una casa de tres o cuatro habitaciones. Hay que tener en cuenta también que ciertos compartimientos no servían como viviendas, sino como almacenes. Mas nuestro propósito no es investigar la funcionalidad o las eventuales agrupaciones de los compartimientos, sino el estudio de las divisiones básicas del esquema urbanístico.

\section{UNA PLANTA ORGANIZADA EN TORNO A UN EJE DE SIMETRÍA NORESTE / SUROESTE}

La calle $\mathrm{R} 2$ fue descubierta durante la última campaña de excavaciones, en septiembre de 1995 , cuando se presentó la oportunidad de abrir un sondeo bajo la calzada de la calle moderna que atraviesa el yacimiento arqueológico por su mitad. Pudimos entonces constatar que el eje de la calle $\mathrm{R} 2$ se encontraba a igual distancia del escarpe del foso noroeste y del escarpe del foso sureste. El eje de la calle $\mathrm{R} 2$ dista $34 \mathrm{~m}$ de la base del paramento de escarpe del foso noroeste, y 33,35 m de la base del talud del foso sureste, cuyo paramento de escarpe y parte del relleno interior han desaparecido como consecuencia de una erosión intensa. Teniendo en cuenta que el retroceso del talud de escarpe del lado sureste bien puede haber alcanzado medio metro, la distancia debía ser originalmente la misma en ambos lados: alrededor de $34 \mathrm{~m}$.

\footnotetext{
${ }^{5}$ Designamos cada compartimiento por medio de una letra que se refiere a la fila en que se sitúa y una cifra que se refiere a su orden en la fila. Por ejemplo, A 1 es el primer compartimiento (partiendo del noreste) de la fila A.
}

La calle R 2 está situada, pues, exactamente en el eje mediano del recinto, dividiéndolo en dos partes iguales. Esta constatación da mucho peso a la hipótesis de una repartición simétrica de las calles y de las manzanas a cada lado de la calle central. Estaríamos ante un urbanismo orientado en el sentido noreste / suroeste y organizado en torno a tres calles paralelas, dos bloques formados cada uno por dos filas de casas y dos filas simples adosadas a las cortinas noroeste y sureste. Los restos de muros que se conservan al este de la calle $\mathrm{R} 2$ apoyan la restitución de una doble fila D-E, simétrica de la doble fila B-C. Un primer lienzo de muro, orientado en sentido perpendicular a la cortina noreste, perteneció probablemente al muro de separación entre D 1 y E 1, mientras que los escasos vestigios de un segundo muro atestiguan la existencia de una pared interna en el compartimiento $\mathrm{E} 1$. Más al este parece lógico restituir una calle $\mathrm{R} 3$, en un sector totalmente destruido por los aterrazamientos modernos.

Este breve resumen de los datos arquitectónicos conocidos demuestra suficientemente que la planta del puerto ibérico se concibió de un modo completamente regular. Destaca no sólo por la disposición geométrica de las manzanas, originadas por calles y murallas trazadas a cordel, sino también por la reproducción sistemática de un solo modelo de casas. Además, veremos a continuación que esta regularidad es el resultado de un planeamiento previo, basado en la utilización de varios módulos métricos que se refieren todos a la misma unidad de medida.

\section{UN ESQUEMA MODULAR}

Los estudios metrológicos aplicados a la arqueología prerromana suelen ser recibidos con cierto recelo. Todos podemos recordar casos en que unos pocos valores métricos, escogidos de forma arbitraria entre muchísimos otros en la planimetría de un yacimiento, han sido esgrimidos como «pruebas» del uso de una supuesta unidad de longitud. Para no caer en tales errores, hemos centrado nuestro análisis en tres elementos arquitectónicos que dan garantías aceptables por su carácter repetitivo y su regularidad : el ancho de los compartimientos, el ancho de las manzanas y de las calles y, en menor grado, las dimensiones de los adobes. Tomados aisladamente, los resultados obtenidos a partir de estos tres elementos no podrían ser considerados como prueba suficiente para apoyar una restitución metrológica. El hecho que queremos destacar es la congruencia de los tres resultados, lo que nos permite afirmar que la unidad de longitud utilizada en La Picola fue un pie cuyo valor oscila entre 29,7 y $30 \mathrm{~cm}$. 


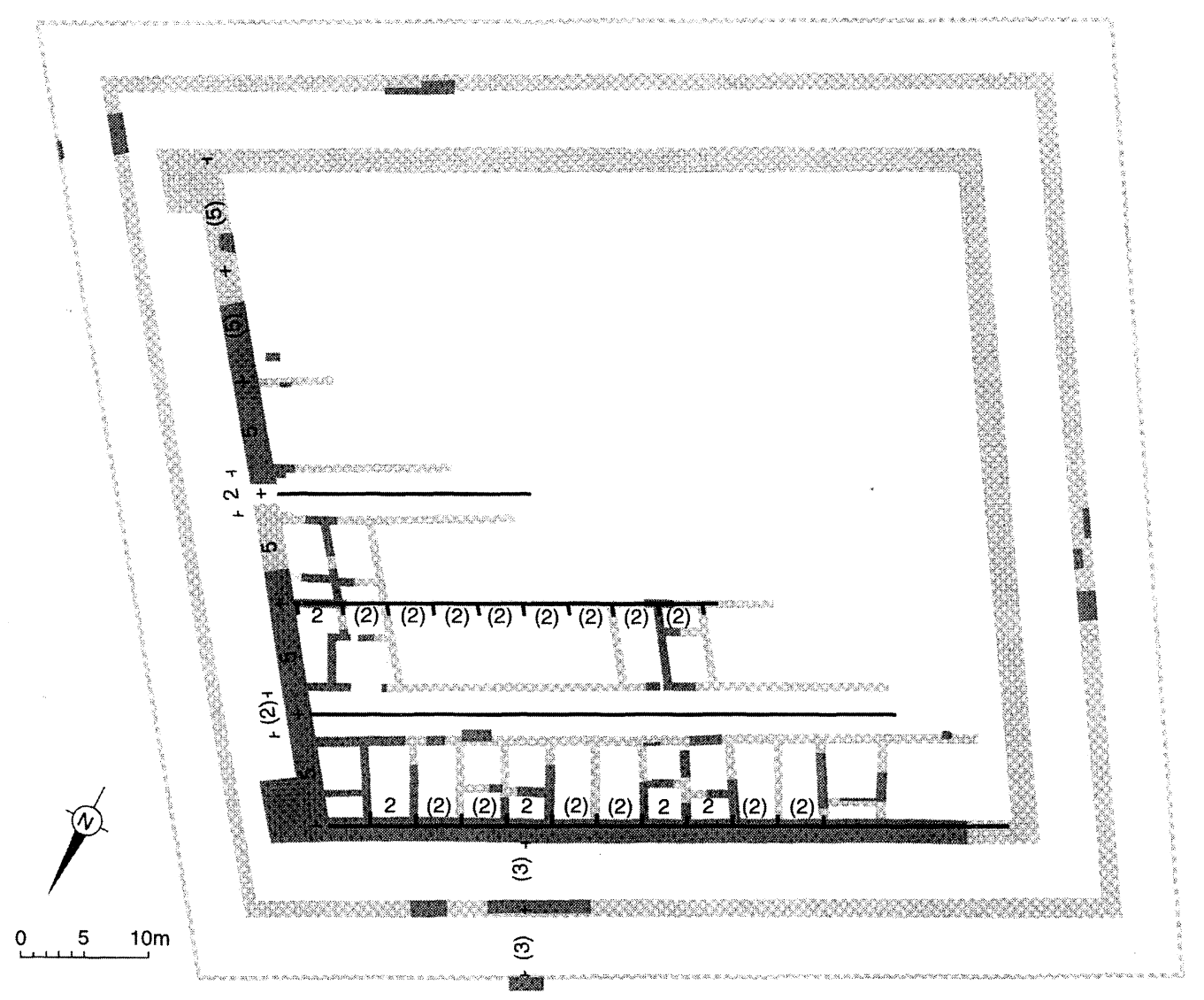

Fig. 4.-Esquema de implantación del módulo 1 (anchura de los compartimientos) y del módulo 2 (anchura de las manzanas y de las calles), en brazas de seis pies.

- Módulo 1: la anchura de los compartimientos (fig. 4)

La planta rectangular de los compartimientos, reproducida en forma casi idéntica a lo largo de las manzanas, es uno de los rasgos más llamativos del urbanismo de La Picola. Cinco compartimientos de la fila A son enteros o casi enteros (A 1, A 2, A 5, A 8 y A 9), y los seis restantes pueden ser fácilmen- te restituidos, dos por dos, en el intervalo de los muros conservados. Contamos además con dos compartimientos del mismo ancho en el extremo noreste de las filas B y C. El siguiente cuadro reúne las medidas de anchura ${ }^{6}$ de todos los compartimientos conocidos, más la anchura de los intervalos que corresponden indudablemente a dos compartimientos contiguos. Las medidas fueron tomadas a ejes de los muros.

\begin{tabular}{|c|c|c|c|c|c|c|c|c|c|c|}
\hline $\mathrm{A} 1 *$ & $\mathrm{~A} 2$ & $\mathrm{~A} 3+\mathrm{A} 4$ & $\mathrm{~A} 5$ & $\mathrm{~A} 6+7$ & $\mathrm{~A} 8$ & $\mathrm{~A} 9$ & $\mathrm{~A} 10+\mathrm{A} 11$ & $A 12 * *$ & $\mathrm{~B} 1 * * *$ & $\mathrm{C} 1$ \\
\hline 3,40 & 3,525 & 7,25 & 3,50 & 7,50 & 3,475 & 3,50 & 7,125 & 4,80 & 3,55 & 3,50 \\
\hline
\end{tabular}

* El compartimiento A 1 tiene una forma ligeramente trapezoidal, por situarse en el ángulo norte que no es ortogonal. Hemos medido su anchura en el centro de su longitud, para conseguir un valor medio.

** El ancho de A 12 rebasa en 1,24 m la anchura media de los demás compartimientos. Está situado en el sector oeste del recinto, que se encuentra muy destruido. No sabemos si el ritmo normal se recuperaba en A 13 .

*** B 1 / B 2 es una casa de planta irregular que se extiende sobre el espacio teórico de dos compartimientos. La división original sólo se conserva en el fondo de la casa, siguiendo la línea del muro de separación entre C 1 y $\mathrm{C} 2$. La anchura indicada corresponde a la habitación trasera de B 1; la habitación delantera es más estrecha.

La serie es sumamente homogénea, si se deja de lado el caso particular de A 12. En estos 13 compartimientos ([A 1 a A 11] + B 1 + C 1), la media aritmé-
${ }^{6}$ No se ha podido tener en cuenta la longitud de los compartimientos debido a que la calle $\mathrm{R} 1$ y la cortina noroeste no son exactamente paralelas, lo que obliga a un aumento progresivo de la longitud de los compartimientos A 1 a A 9. 
tica de las anchuras es de 3,563 $\mathrm{m}$. La diferencia media absoluta es de $7,6 \mathrm{~cm}$, lo que confiere a la cifra obtenida una precisión estadística bastante buena.

Esta anchura media está confirmada en la fila B por la distancia de $28,55 \mathrm{~m}$ que se mide entre el paramento interno de la muralla y la pared sur de B 8. Aunque no conocemos la organización real de los compartimientos B 2 a B 8, esta distancia corresponde muy exactamente, con una diferencia inferior al centímetro, a la media que acabamos de obtener $(28,55 \mathrm{~m}: 8=3,568)$.

Se confirma, pues, que la división de los bloques en compartimientos se realizó en base a un módulo que medía entre 3,56 y $3,57 \mathrm{~m}$. Esta cifra se puede traducir en 12 pies de un valor cercano a $29,7 \mathrm{~cm}$ $(12 \times 29,7=3,564)$.

El caso del compartimiento A 12 puede ser reevaluado a la luz de estos resultados. Su anchura es de $4,80 \mathrm{~m}$, lo que equivale muy precisamente a 16 pies de $30 \mathrm{~cm}$, es decir el módulo 1 aumentado en su tercera parte $(12+4$ pies). Ignoramos la causa de este ensanchamiento, pero no hay duda de que el compartimiento A 12 refleja, como los demás, un sistema metrológico basado en un pie cercano a los $30 \mathrm{~cm}$.

\section{- Módulo 2 : la distancia entre los ejes de las ca-} lles y de las manzanas (fig. 4)

Se puede observar, en el sector norte del recinto, que la cortina noroeste, las calles R 1 y R 2 y la manzana B-C están separados por una distancia constante que ronda los nueve metros.

- Desde el eje del bloque B-C hasta el eje de R 1: $9,00 \mathrm{~m}$.

- Desde el eje del bloque B-C hasta el eje de R $2: 8,80 \mathrm{~m}$

- Desde el eje de R 1 hasta el eje de la cortina noroeste : $9,25 \mathrm{~m}$.

Estamos en presencia de un segundo módulo, tres veces repetido a lo largo de la cortina noreste. Constituía el armazón de la organización interior del poblado. Los mismos elementos se repetían muy probablemente, en orden inverso, en la mitad este del recinto.

No obstante, lo que sabemos de este segundo módulo no nos permite precisar el valor métrico de la unidad de longitud utilizada en La Picola. Tres medidas no constituyen una base estadística suficiente. Lo único que se puede proponer es un valor aproximado del pie contenido en el módulo 2 , sin posibilidad de ponderación estadística. La distancia de 27,05 m que se mide entre el eje de R 2 y el eje de la cortina noroeste (es decir, tres veces el módu- lo 2) equivale a 90 pies de $30,05 \mathrm{~cm}$. Ahora bien, si tenemos en cuenta solamente la distancia entre el eje de R 1 y el eje de R 2, llegamos a 60 pies de un valor algo inferior: $29,66 \mathrm{~cm}$. En todo caso, no nos alejamos mucho del pie deducido del módulo 1, ya que en ambos módulos el pie restituido está muy cerca de los $30 \mathrm{~cm}$.

Una constatación más importante es que el módulo 2, al igual que el módulo 1, puede ser dividido en brazas. La braza griega, llamada orgüia, valía 6 pies o $4 \operatorname{codos}^{7}$; su uso era frecuente en arquitectura por un motivo práctico: una cuerda dividida en brazas era más manejable que un pie de metal o de madera para medir construcciones de cierta amplitud. A. Roth-Congès, seguida por $\mathrm{H}$. Tréziny ${ }^{8}$, ha demostrado de manera convincente que la braza de seis pies fue la principal unidad de medida en varias construcciones helenísticas de la Galia meridional, tanto en el área propiamente griega (en Marsella durante el siglo II a.C.) como en el hinterland indígena más o menos helenizado (en Saint-Blaise, en Glanum y en Entremont) ${ }^{9}$. Lo mismo puede decirse de La Picola, donde los dos módulos que acabamos de definir se descomponen de la manera siguiente:

Módulo $1=2$ brazas $=12$ pies de $29,7 \mathrm{~cm}$.

Módulo $2=5$ brazas $=30$ pies de unos $30 \mathrm{~cm}$.

Las 5 brazas del módulo 2 se dividen teóricamente en $4+1$, longitud de un compartimiento más la mitad del ancho de una calle. En la práctica, sólo la calle R 2 se conforma con este esquema básico. Su anchura de 3,55 m corresponde exactamente al módulo 1 ( 2 brazas). En cambio, la longitud de los compartimientos del bloque B-C $(6,95$ a $7,10 \mathrm{~m}$ entre el eje medianero del bloque y el borde de la ca1le) es un poco inferior a 4 brazas, mientras que la anchura de la calle $R 1$ rebasa claramente las 2 brazas (de 3,80 a $3,96 \mathrm{~m}$ ).

En definitiva, el descubrimiento de esta trama geométrica nos permite reconstruir con cierta precisión las fases de la construcción en la mitad noreste del yacimiento :

\footnotetext{
${ }^{7}$ Heródoto, 2, 149.

${ }^{8}$ A. ROTH-CONGÈs, «Glanum préromaine: recherches sur la métrologie et ses applications dans l'urbanisme et l'architecture», Revue archéologique de Narbonnaise, 18, 1985, 189-220; H. TRÉziNY, «Métrologie, architecture et urbanisme dans le monde massaliète», ibid., 22, 1989, 1-46.

${ }^{9}$ Ello no impide una gran diversidad del pie que se deduce de la braza utilizada en cada yacimiento: $27,5 \mathrm{~cm}$ en Saint-Blaise, $28,1 \mathrm{~cm}$ en Entremont, $34,8 \mathrm{~cm}$ en la fortificación helenística de Marsella.
} 
1.1. Se fija la longitud de la cortina noreste en 30 brazas hasta su intersección con los ejes de las cortinas noroeste y sureste.

1.2. Esta línea de 30 brazas se divide en segmentos de 5 brazas (módulo 2). Cada división coincide con el eje de una calle (a 5, 15 y 25 brazas del ángulo norte) ${ }^{10}$ o con el eje de una manzana constituida por dos filas de casas (B-C a 10 brazas, D-E a 20 brazas del ángulo norte). Vemos, pues, que al módulo de 5 brazas se superpone un módulo de 10 brazas, jalonado por los ejes de manzanas. Se vislumbra, en este caso, la posible utilización de otra medida de longitud griega, la cádena o hamma, que valía 10 brazas o 40 codos ${ }^{11}$.

1.3. La línea de fachada de las manzanas están trazadas paralelamente a las cortinas noroeste y sureste, a una braza (aproximadamente) del eje de las calles. Pero esta operación se realiza con menor esmero que las precedentes. Además, el grosor de la muralla (1 braza) obliga a reducir la anchura de la fila A con respecto a las filas B-C y D-E.

2. Las manzanas reciben divisiones internas, de dos en dos brazas (módulo 1), creándose así una serie de compartimientos destinados a la vivienda o al almacenamiento.

\section{LOS ADOBES}

Los adobes encontrados in situ en el alzado de varios muros de la fila B fueron estudiados por Claire-Anne de Chazelles ${ }^{12}$. A pesar del tamaño reducido de la muestra (veinte adobes, algunos de ellos rotos), fue posible evidenciar un módulo entero y un medio módulo.

- Módulo entero: longitud 37-38 cm (media calculada sobre 4 adobes: $37,5 \mathrm{~cm}$ ), anchura $29-32 \mathrm{~cm}$ (media sobre 3 adobes: $30 \mathrm{~cm}$ ), espesor $8,5 \mathrm{~cm}$.

- Medio módulo: longitud 29-32 cm (media sobre 4 adobes: $30,2 \mathrm{~cm}$ ), anchura 18-23 cm (media sobre 13 adobes: $20 \mathrm{~cm}$ ), espesor $8,5 \mathrm{~cm}$.

La anchura del módulo entero y la longitud del medio módulo equivalen a un pie de unos $30 \mathrm{~cm}( \pm$ $1 \mathrm{~cm}$ ). Es la misma unidad de medida que hemos encontrado en la planta de las casas y calles.

Podemos constatar además que la longitud del módulo entero equivale a cinco cuartos de pie $(30 \mathrm{x}$ $1,25=37,5)$. Ahora bien, esta medida es justamente la que define el ladrillo pentadoron, cuya longitud

\footnotetext{
${ }^{10}$ Sólo se han excavado dos calles. La existencia de la tercera, en el lado este, se deduce por simetría, pero no es más que una hipótesis.

${ }^{11}$ Herón, Geometrica, 23, 14.

${ }^{12}$ Estudio incluido en Roulllard et al., en prensa (cit. n. 2).
}

era de cinco palmas según Vitruvio ${ }^{13}$. Se conocen ejemplares de pentadoron en muchos yacimientos griegos a partir de la época clásica, inclusive en la Magna Grecia ${ }^{14}$. Sin embargo, puede tratarse de una coincidencia, y la hipótesis de una tradición local no es menos aceptable. Un módulo de 40 × 30 $\mathrm{cm}$, muy próximo a nuestro módulo 3 , está atestiguado en varios yacimientos ibéricos de la Segunda Edad del Hierro ${ }^{15}$; además, en dos casos por lo menos, a este módulo se añade un medio módulo que corresponde exactamente al de La Picola: $30 \mathrm{x}$ $20 \mathrm{~cm}^{16}$.

\section{PROBLEMAS NO RESUELTOS}

Los resultados que acabamos de presentar no deben ocultar las incertidumbres y las muchas incógnitas que persisten. No conocemos los límites del recinto por el lado del mar (al suroeste), excepto en dos sondeos profundos en los que se descubrió un breve fragmento del escarpe del foso. No podemos determinar, por consiguiente, la longitud exacta de las cortinas noroeste y sureste. No sabemos si existió o no un segundo eje de simetría en el sentido noreste / suroeste, puesto que no ha aparecido ningún resto de una calle perpendicular a $R 1$ y a R 2 .

El único ángulo conservado del recinto es el norte. Se trata de un ángulo obtuso (110 grados). No sabemos por qué los constructores eligieron este ángulo, ya que el terreno es perfectamente llano. Sólo podemos constatar que esta particularidad acarreó complicaciones en el trazado interior del poblado, puesto que fue necesario elegir entre dos orientaciones posibles, no sin vacilaciones. Así, la longitud de los compartimientos de la fila A aumenta progresivamente de norte a sur; las calles corren aproximadamente paralelas a la cortina noroeste, pero no son perpendiculares a la cortina noreste; una parte de los muros de los compartimientos son paralelos a la cortina noreste (C1/C2, B8/B9), mientras que otra parte son perpendiculares a la cortina

\footnotetext{
${ }^{13}$ Vitruvio, II, 3, 3. La palma griega equivalía a la cuarta parte de un pie.

${ }^{14}$ Véase la reciente síntesis de V. Righini, «Materiali e tecniche da costruzione in età preromana e romana», en Storia di Ravenna, I. L'evo antico, Ravenna, 1990, 271-272.

${ }^{15}$ Véase L. Abad y F. SALA, El poblado ibérico de El Oral (San Fulgencio, Alicante), Valencia, 1993, 195-197, para el Sureste y el Levante, y J.A. Asensio Esteban, La ciudad en el mundo prerromano en Aragón, Zaragoza, 1995, 385-390, para el valle del Ebro.

${ }^{16}$ En Puntal dels Llops (Olocau, Valencia) y en El Amarejo (Bonete, Albacete), en niveles fechados en los siglos iv y III a.C.
} 
noroeste (B1/B2, A2/A3), y una tercera parte adopta una orientación intermedia.

La fortificación no ha proporcionado datos metrológicos muy seguros, debido a las importantes variaciones observadas en las dimensiones de cada unos de sus elementos. No obstante, las medidas registradas parecen compatibles con un sistema metrológico basado en brazas. La anchura de la cortina $(1,65$ a $1,80 \mathrm{~m}$ ) equivale a una braza de seis pies, con un pie ligeramente inferior a $30 \mathrm{~cm}$. Es de notar, a este respecto, que la primera muralla de Olbia en Liguria, del siglo IV a.C., medía también una braza de ancho ${ }^{17}$. La anchura del glacis (5 a 5,5 m) y del foso (5 a $6 \mathrm{~m}$ al nivel del suelo) pueden corresponder a 3 brazas, pero en este caso la variación es demasiado grande como para determinar el valor del pie. Por último, podemos señalar que la torre angular mide de ancho $4,75 \mathrm{~m}$, lo que equivale a 16 pies de $29,7 \mathrm{~cm}$.

\section{UN MODELO ARQUITECTÓNICO GRIEGO PARA UN ASENTAMIENTO INDÍGENA}

Acabamos de ver que todo el programa arquitectónico de La Picola, desde los materiales de construcción hasta el planeamiento general, se basó en un pie de 29,7 a $30 \mathrm{~cm}$ y que la aplicación de esta unidad de medida se hizo por medio de una braza de seis pies.

A este respecto, La Picola constituye un unicum en la Iberia del siglo v a.C. Dos generaciones antes, en la misma región, el poblado de El Oral presenta un esquema racional y complejo, entroncado en las tradiciones del urbanismo orientalizante del sur de la Península Ibérica ${ }^{18}$, quedando empero muy alejado de la rígida trama modular que se observa en La Picola. Por otra parte, lo poco que se conoce de las colonias fenicias de España no sugiere una división geométrica del área habitada.

Queda la posibilidad de un origen griego. Muy poco se sabe de las fortificaciones de Emporion durante el siglo $\mathrm{v}$ a.C., pero se conserva un buen tramo del lado sur de la muralla griega del siglo IV, con dos torres rectangulares unidas por una cortina recta. A tenor de los datos publicados por Enric Sanmartí ${ }^{19}$, se vislumbra la utilización de un pie li-

\footnotetext{
${ }^{17}$ Según las observaciones de J. Benoit en el lado sur, la cortina primitiva de Olbia tenía un grosor de 1,52 a $1,63 \mathrm{~m}$, lo que correspondería a seis pies en la metrología «menor» atestiguada en Olbia (TRÉZINY, cit. n. 8, p. 22).

${ }^{18}$ Abad y Sala, cit. n. 15.

${ }^{19}$ E. Sanmartí, P. Castanyer y J. Tremoleda, «La secuencia histórico-topográfica de las murallas del sector meridional de Emporion», Madrider Mitteilungen, 29, 1988, p. 191200 (especialmente fig. 5 y lám. 14).
}

geramente inferior a $30 \mathrm{~cm}$ en la construcción de esta muralla. La cortina mide $26,8 \mathrm{~m}=90 \times 29,8$ $\mathrm{cm}=15$ brazas; el lado mayor de la torre mejor conservada mide $9,5 \mathrm{~m}=32 \times 29,7 \mathrm{~cm}$ y su lado menor (hasta la cara interna de la muralla) mide 7,2 $\mathrm{m}=24 \times 30 \mathrm{~cm}=4$ brazas $^{20}$.

A pocos kilómetros de la colonia griega, el yacimiento indígena del Puig de Sant Andreu (Ullastret, Girona) posee desde el inicio del siglo V a.C. una fortificación que sigue también las pautas de un esquema modular rigurosamente planificado. Los elementos de la fortificación están casi todos calculados en base a un módulo de unos 4,75 m (es decir, muy probablemente, 16 pies de $29,5 \pm 0,2 \mathrm{~cm}$ ). El diámetro de las torres circulares equivale por ejemplo a 2 módulos, los lados de la gran torre del Istmo a 3 módulos, las cortinas situadas entre las torres circulares a 6 módulos, varios segmentos de muralla separados por retranqueos, esquinas o poternas a 3, 4, 6, 7, 10 o 13 módulos ${ }^{21}$.

A pesar de las diferencias de tamaño, aparejo y funciones, las plantas de los asentamientos de Emporion, Ullastret y La Picola presentan importantes semejanzas. Todas se basan en un pie de 29,5/30 cm, idéntico al pie jonio-ático que tuvo una amplia difusión en el mundo griego y particularmente en varios yacimientos foceos del Oeste. Henri Tréziny, en su trabajo pionero sobre la metrología massaliota ${ }^{22}$, ha presentado varios indicios que hablan a favor de la existencia en Marsella, en época arcaica, pero también en Velia entre los siglos v y III a.C. ${ }^{23}$, de un pie que medía alrededor de $30 \mathrm{~cm}$. Es más: la braza de 6 pies que se utilizó en Emporion y La Picola es una medida típicamente griega, documentada, como hemos visto, en varios programas arquitectónicos de cierta envergadura del territorio de Massalia y de su área de influencia más cercana. Por otra parte, se reconoce el mismo módulo de 16 pies en las torres de los tres yacimientos analizados: 2 x 16' en el diámetro de las torres circulares de Ullastret, 2 x $16^{\prime}$ en la anchura de la torre rectangular de Emporion, y 16' en la anchura de la torre de La Picola.

${ }^{20}$ Detalle del análisis en P. MoRet, «'Rostros de piedra'. Sobre la racionalidad del proyecto arquitectónico de las fortificaciones urbanas ibéricas», en Los iberos príncipes de Occidente (Congreso Internacional, Barcelona, 12-14 de marzo de 1998), Barcelona, Fundación la Caixa, 1998, en prensa.

${ }^{21}$ Moret, cit. n. 20.

${ }^{22}$ TrÉzinY, cit. n. 8.

${ }^{23}$ A juzgar por el módulo de los ladrillos de barro cocido y las medidas de varios monumentos. No ha sido posible sacar datos concluyentes del análisis metrológico del urbanismo ( $c f$. F. KRINZINGER, «Intorno alla pianta di Velia», en Velia - Studi e ricerche, a cura di G. Greco \& F. Krinzinger, Modena, 1994, 19-54). 
Los datos obtenidos, tanto en Ullastret como en Santa Pola, abogan claramente por un modelo metrológico griego, probablemente originado en el área focea occidental. Sin embargo, las cinco hectáreas de área superficial y la disposición irregular de las calles del oppidum amurallado de Ullastret no se pueden equiparar con el diminuto tamaño y la trama geométrica de La Picola. No se conoce, hasta la fecha, la planta de ningun fortín costero o factoría comercial griega del siglo v a.C. Sin embargo, ciertas construcciones de la centuria siguiente, entre las que destaca el epiteichisma de Olbia, al oeste de Marsella ${ }^{24}$, cuyo plano ortogonal recuerda en varios aspectos el de La Picola, pueden darnos una idea aproximada de este tipo de establecimientos fortificados de pequeño tamaño que debían existir en la zona de influencia de las más importantes colonias griegas del Mediterráneo occidental.

Esto no quiere decir que La Picola fuera un asentamiento griego. El material cerámico encontrado en el transcurso de las excavaciones obliga a descartar esta interpretación. La cerámica ática sólo alcanza el $10 \%$ de la vajilla (exceptuando las ánforas), mientras que todo lo demás corresponde a producciones ibéricas, y sólo el 2,5\% de las ánforas son griegas ${ }^{25}$. A título de comparación, bastará recordar que en Emporion la cerámica ática alcanza el $72 \%$ de la vajilla fina de mesa entre 450 y 400 a.C. y el

${ }^{24}$ La fundación del fortín militar de Olbia, hacia 330, coincide con el abandono de La Picola (J. CouPRY, «Les fortifications d'Olbia de Ligurie. Propositions, questions», en $\mathrm{La}$ fortification dans l'histoire du monde grec, Paris, 1986, 389399). Cabe señalar varias semejanzas entre ambas construcciones, a pesar de la diferencia de tamaño (las cortinas de Olbia son tres veces más largas que las de La Picola). La primera muralla de Olbia comparte con la de La Picola el mismo aparejo irregular, con alzado de adobes, y tiene el mismo grosor. La planta del recinto de Olbia es cuadrada y sus manzanas se insertan, como en La Picola, en un esquema modular y simétrico.

${ }^{25}$ Un análisis pormenorizado de la cerámica está realizado por Eric Gailledrat y Pierre Rouillard en RouILLARD et al., en prensa (cit. n. 2). Los porcentajes están calculados en función del número mínimo de individuos.
$92 \%$ entre 400 y $375^{26}$. En Olbia, en el último tercio del siglo Iv, la totalidad de la vajilla de mesa viene del Ática o de Marsella, y la vajilla de cocina también es griega ${ }^{27}$.

Hasta en la arquitectura, ciertos detalles parecen originarse en una tradición indígena local. Por ejemplo, la disposición poco corriente de la torre del ángulo norte, con un solo lado saliente, recuerda la torre del poblado ibérico de El Oral ${ }^{28}$. Los rasgos indígenas contrapesan, pues, los influjos griegos.

La explicación de este balance contradictorio, según se mira hacia el urbanismo o hacia los vestigios de la vida cotidiana, debe buscarse en el contexto regional de La Picola. Un asentamiento tan pequeño no hubiese podido nacer y no hubiese podido subsistir fuera del control directo de Elche, la ciudad entonces más potente de la región, situada a pocos kilómetros tierra adentro. Lejos de ser un enclave griego, el puerto de La Picola debe ser considerado como una factoría marítima indígena, una avanzadilla de Elche abierta al comercio griego, diríamos incluso creada para el comercio griego. $\mathrm{Pa}$ rece lícito suponer -aunque la confirmación de tal hipótesis es imposible- que la construcción de la factoría fuera confiada a un griego y, en todo caso, no hay duda de que La Picola fue regularmente visitada por comerciantes griegos. El único hecho seguro, empero, es que la mayoría de sus habitantes eran iberos. A igual distancia del poblado indígena tradicional y del enclave colonial, La Picola se presenta, en suma, como la plasmación híbrida de un contacto prolongado entre dos culturas mediterráneas, el reflejo en tierra ibérica de la arquitectura griega occidental.

\footnotetext{
${ }^{26}$ E. SANMARTí, «Massalia et Emporion: une origine commune, deux destins différents», en Marseille grecque et la Gaule (Etudes Massaliètes, 3), Lattes, 1992, 35.

${ }^{27} \mathrm{M}$. BATs, Vaisselle et alimentation à Olbia de Provence (v. 350 - v. 50 av. J.-C.). Modèles culturels et catégories céramiques (RANarb., Supplément 18), Paris, 1988, 201-202 y

${ }^{28}$ Véase Abad y Sala, cit. n. 15
} 226 